\title{
Extended Use of Vettath's Anastomotic Obturator (VAO)
}

\author{
Vettath Prabhakaran Murali", Ismail E. Thazhkuni, Kannan A. Vellachamy \\ Malabar Institute of Medical Sciences, Kozhikode, India \\ E-mail: *mvettathcts@hotmail.com
}

Received June 8, 2011; revised September 21, 2011; accepted October 26, 2011

\begin{abstract}
Stroke rate in redo coronary artery bypass surgery has remained a stumbling block, where the aorta is used as origin for the top end of the vein graft. Avoiding the side-clamp on these redo aortas by using the Vettath's Anastomotic Obturator (VAO) technique of anatomosing the vein graft could bring this down. We have also been able to use this technique in combined aortic valve replacement and CABG. These two situations where the VAO is used are elucidated here.
\end{abstract}

Keywords: Anastomosis, Coronary, Redo CABG, VAO

\section{Introduction}

Redo Coronary artery bypass grafting (CABG) has become a common procedure in many surgical units. Though it can be safely and effectively performed, stroke rate in Redo CABG has been high in literature. Proximal anastomotic devices have been found to reduce stroke rates in CABG's $[1,2]$.

Vettath's Anastomotic Obturator (VAO) is a proximal anastomotic device developed by the author (MPV). In our center, it has been used in more than 500 proximal top end anastamosis of coronary vein grafts over the past eight years. We have been able to avoid the use of aortic side clamp on diseased and thickened redo aortas by employing this device. This avoids the release of calcium from the aorta and there by avoiding stroke. Extending its use to redo CABG as well as in aortic valve procedure and $C A B G$ is highlighted here.

\section{Technique}

VAO can be employed for both on pump and off pump Redo-CABG. The approach is through median sternotomy. It is universally observed that the proximal stump of even totally occluded vein grafts are soft and offer an ideal site for placing top ends of new vein grafts. These sites are identified.

After completing distal anastomoses, a 3-0-polyprolene purse-string suture is placed around the intended site of top end anastomosis and it is snared. A No.11 knife is used to make a stab incision in the center of the pursestring suture. This hole is occluded with the left index finger and punched out using a $4.5 \mathrm{~mm}$ aortic punch. This site is again covered with left index finger to prevent bleeding. Then the VAO (Figure 1) is inserted into the punch hole and the snare is tightened just enough to prevent bleeding. The proximal end of the vein graft is then sutured to the new punch hole using 5-0-polypropylene suture using the technique already described [4] The same technique could be used to perform 2 or 3 top ends (Figure 2).

Another similar circumstance where a side clamp on aorta is better avoided is in combined aortic valve surgery and CABG. Here, the presence of calcium on aortic wall or the need to avoid crushing the aortic suture line necessitates the use of VAO, if the surgeon intends to perform the top end after release of the cross clamp for want of space in short ascending aortic patients. The proximal end of vein graft is connected to the aorta using the aforementioned technique after removal of crossclamp.

\section{Discussion}

The need to put a side clamp on aorta for proximal anastomoses of free grafts (either veins or arteries) has been a stumbling block in atherosclerotic or calcified aortas. Redo CABGs where ascending aorta is often thickened, plaquey and stuck to the adjacent tissue is one such instance. Similarly, the desire to avoid aortic side clamp after closure of aortotomy (to avoid crushing of suture line) in combined aortic valve and CABG procedures also necessitates the use of alternative devices for proximal anastomosis. VAO is an anastomotic device, 


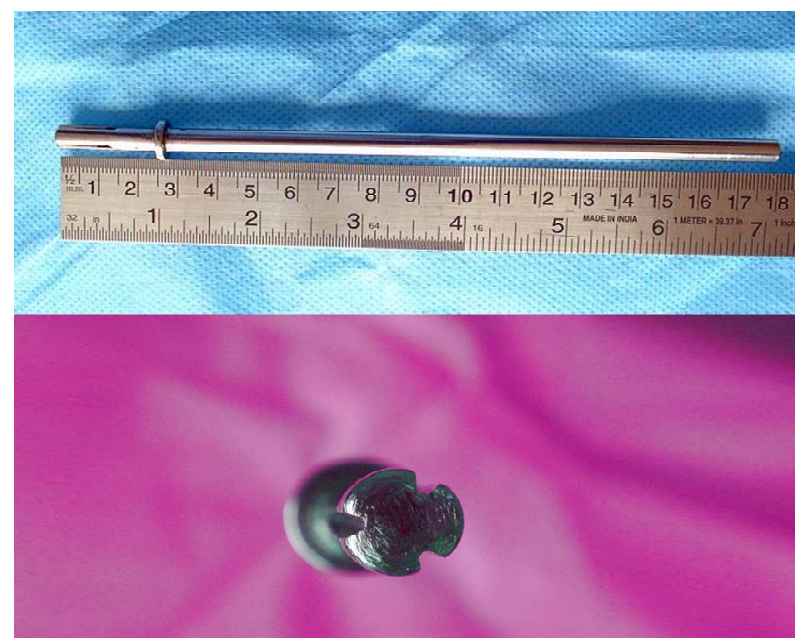

Figure 1. VAO-different views.

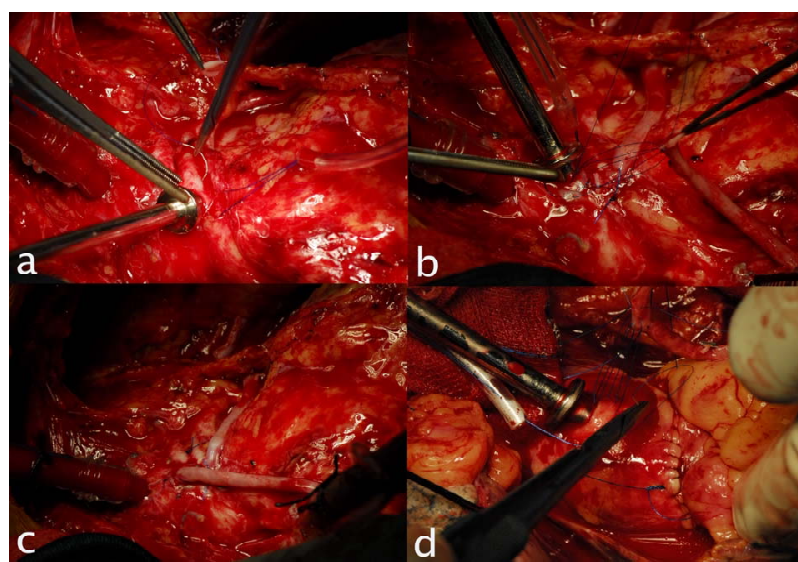

Figure 2. Operative photograph showing: (a) commencing anastomosis using VAO; (b) anastomosis in progress; (c) after completion of proximal anastomosis; (d) combined aortic surgery and CABG using VAO. which carries all the advantages of a proximal anastomotic device, in the meantime enabling the surgeon to perform a good handsewn anstomosis under vision. The advantage of this device is its reusablity and its affordability in the third world countries.

The author has used this device to perform his proximal anastomosis in 20 redo CABG's with no neurological deficit. This proximal anastomotic device can also be potentially used in short and thickened aortas and in combined procedures (like Aortic valve replacement/ repair + CABG). The advantage of this device is its simple design, cost effectiveness and the ease of constructing a good hand sewn anastomosis maintaining good patency as mentioned in our previous reports [4].

\section{References}

[1] S. F. Aranki, P. S. Shekar, A. Ehsan, M. Byrne-Taft and G. S. Couper, "Evaluation of the Enclose Proximal Anastomosis Device in Coronary Artery Bypass Grafting,' The Annals of Thoracic Surgery, Vol. 80, No. 3, 2005, pp. 1091-1095.

[2] R. S. Boova, C. Trace and B. G. Leshnower, "Initial Experience with Enclose Proximal Anastomotic Device During off-Pump Coronary Artery Bypass: An Alternative to Aortic Side Clamping," Heart Surgery Forum, Vol. 19, No. 2, 2006, pp. E607-E611.

[3] M. P Vettath, "Vettath's Anastamotic Obturator: A Simple Proximal Anastamotic Device," The Heart Surgery Forum, 2003, Vol. 6, No. 5, Article ID: 73305. www.hsforum.com/vol6/issue5/2003-73305.html

[4] M. P Vettath, A. V. Kannan, C. S. Sheen Peeceeyen, A. K. Baburajan, A. Vahab and M. P. Sujith, "Vettath's Anastamotic Obturator-Our Experience Of 269 Proximal Anastamosis," Heart, Lung and Circulation, Vol. 13, No. 3, 2004, pp. 288-290. doi:10.1016/j.hlc.2004.02.019 